\title{
Verdrängung und Vernichtung der anspruchsvollen Gehölze am Beginn der letzten Eiszeit und die Korrelation der Frühwürm-Interstadiale in Mittel- und Nordeuropa
}

\author{
Max Welten *)
}

Upper Pleistocene (Würm interstadial), pollen diagram, Angiospermae, Coniferales, extent, extinction, paleoclimate, age, C-14-dating, correlation (of Early Würm interstadials), Central Europe

K u rzfassung: Angenähert vollständige Profile von organischen oder sekundärpollenfreien mineralischen Sedimenten über die Zeit des R/W-Interglazials und der Frühwürm-Interstadiale bieten die Möglichkeit, die allmähliche Verdrängung und das mehrmalige Wiederauftreten der anspruchsvollen Gehölze Mittel- und Nordeuropas zu studieren. Vier Pollenprofile wurden auf gleichen Maßstab umgezeichnet. Ihr Vergleich liefert eine überraschende Übereinstimmung der Erscheinungen und einen Weg zur Korrelierung der Frühwürm-Interstadiale.

\section{[Displacement and Extermination of Warmth-Demanding Elements of European Forests during Early Weichselian Glacial and Correlation of Early Weichselian Interstadials]}

A bstract: Complete series of organic material from R/W-Interglacial and subsequent Early Weichselian Interstadials near the Alps give the possibility of studying the displacement and extermination of warmth demanding elements of Middle European forests. They represent by no means the mere reflected image of the events of reimmigration of Late- and Postglacial times. Beyond this they form a means for correlation of Early Weichselian Interstadials.

[Déplacement et suppression des éléments thermophiles des forêts de l'Europe Centrale pendant les interstades du Eowurm et corrélation de ces interstades]

$\mathrm{R}$ é $s \mathrm{u}$ mé : Lors du commencement de la dernière glaciation les éléments thermophiles des forêts de l'Europe Centrale furent refoulés et à la fin anéantis en plusieurs étapes stadiaires et interstadiaires qu'on arrive-t-aujourd'hui à connaître exactement. Une comparaison de ces évènements sur un transect Europe du Nord/Alpes fait sortir ces étapes de régression qui n'ont nullepart l'aspect négatif de la réimmigration tardi- et postglaciaire. Ceux-ci aident à corréler les Interstadiaires du Early Weichselian (Eo-Würm).

\section{Einleitung}

In den klassischen Ländern der biostratigraphischen Interglazial- und Interstadialforschung Nordeuropas, insbesondere in den Niederlanden, Dänemark und Norddeutschland haben in den letzten Jahren Zagwijn \& Paepe (1968) und Menke \& Behre (1973) Übersichten veröffentlicht, die den Stand der Forschung in Nordeuropa darstellen. Seither haben in Mitteleuropa Woillard (1975), Grüger (1979a), der Verfasser (Welten 1976, 1981 [im Druck]) und Klaus (1975), 1981 [im Druck]) lange Profile aus dem Bereich der alpinen Vergletscherung über das R/W-Interglazial und das Frühwürm bearbeitet, die einen Vergleich herausfordern.

GRÜGER (1979b) hat sein schönes Diagramm vom Samerberg in Oberbayern den nordeuropäischen Ergebnissen und dem Diagramm von Grande Pile von WoIllard zugeordnet unter Berücksichtigung der allgemeinen Entwicklungstendenzen, insbesondere der

*) Anschrift des Verfassers: Prof. em. Dr. M. W e 1 t e n, Systematisch-geobotanisches Institut der Universität Bern, Altenbergrain 21, CH-3013 Bern (Schweiz), privat: Hohliebestraße 14, CH-3028 Spiegel-Bern. 
Zweiteilung des Brörup-Interstadials i.w.S. Sein Hauptaugenmerk war auf die Korrelation des R/W-Interglazials mit dem Eem-Interglazial des Nordens gerichtet.

Der Verfasser wurde durch die Bearbeitung und Auswertung eines fast $4 \mathrm{~m}$ langen Profils von lockerem (vom Gletscher nicht überfahrenem) Torf auf dem Sulzberg bei Baden-Wettingen, $3 \mathrm{~km}$ vor den Würm-Maximalmoränen von Killwangen, durch das systematische Auftreten von deutlichen Gipfelchen anspruchsvoller Gehölze in den Frühwürm-Interstadialen auf die Möglichkeit hingewiesen, die Frühw ürm-Interstadialphasen in Europa mit Hilfeder pulsierenden Vorstöße 'anspruchsvoller Arten zu vergleichen und zu korrelieren.

Wir brauchen uns primär nicht darum zu kümmern, ob es sich um W i e d e r b e s i e d lung der Lokalitäten oder um Pollenniederschlag a u Fernflug handelt.

\section{Vergleichsobjekte}

Für den Vergleich wurden Objekte ausgewählt, die an das letzte Interglazial anschließen, möglichst vollständig sind, mindestens 2-3 Interstadiale aufweisen, und die von sekundärer Polleneinschwemmung \pm frei sind.

In Mitteleuropa entsprechen folgende Frühwürm-Interstadialfolgen diesen Bedingungen (* für unsern Diagrammvergleich verwendet):

*Grande Pile (Woillard 1975), westlich der Vogesen

*Samerberg (GRüGER 1979), Oberbayern

*Sulzberg-Baden (WELTEN 1981), unterhalb Killwangen bei Zürich

Dürnten (WELTEN 1981), Zürcher Oberland

Uster (Welten 1981), Glatt-Tal, Kt. Zürich

Mondsee (Klaus 1981), Salzburg, Österreich

In Nordeuropa ist die Mehrzahl der Abfolgen unvollständig. Aufschlußreich und an Eem anschließend sind:

Rodebaek und Brörup (ANDERSEN, S. T. 1961), Dänemark

*Amersfoort (und Wanssum) (ZAGwijn 1961), Niederlande

*Odderade (Averdieck 1967), Schleswig-Holstein, N-Deutschland

Osterwanna (BEHRE 1974), Niedersachsen, N-Deutschland.

\section{Umzeichnung der Vergleichsdiagramme}

Der Vergleich so vieler Erscheinungen an so verschiedenen Lokalitäten läßt sich anschaulich nur graphisch durchführen. Die Diagramme wurden deshalb umgezeichnet in

a) ein Kurven-Totaldiagramm rechts mit Betula, Pinus, Picea und NBP-Darstellung (unter Weglassung der niedrigen Werte anspruchsvoller Gehölze, jedoch mit Eintragung auszeichnender lokaler Dominanzen)

b) und Silhouetten-Darstellungen der uns besonders interessierenden anspruchsvollen Gehölze links davon. Den in Europa so unterschiedlichen Prozentwerten werden wir gerecht durch zwei Darstellungsmaßstäbe (schwarze Silhouetten = große Prozentwerte, weiße Silhouetten $=$ fünf mal kleinere Werte).

Rechts fügen wir in ähnlichem Doppelmaßstab Artemisia an und versuchen die Hochmoorentwicklung des Profils durch Angabe der Sphagnum-Prozente anzudeuten. Die Zahl der ausgezählten Pollen läßt die statistische Ausgeglichenheit oder die Tendenz zu Schwankungen der oft kleinen Prozentwerte verstehen. Die Materialangaben in der 2. Spalte sind pauschal gehalten. 




Abb. 1: Lage der wichtigsten Eem-Frühwürm-Profile (in Klammer die Nummer der zitierten Publikation)

$1=$ Amersfoort (23), 2a = Odderade (2), 2b = Rederstall (12, 17), $3=$ Grande Pile (21, 22), $4=$ Sulzberg-Baden (20), $5=$ Samerberg $(7,8), 6=$ Mondsee (9, Frühwürm nicht publiziert), $7=$ Wanssum (23), $8=$ Osterwanna (3), $9=$ Oerel $(14,16), 10=$ Geesthacht $(15), 11=$ Keller $(12,17), 12=$ Loopstedt (15), 13 = Brörup Hotel Bog (1), $14=$ Rodebaek (1), $15=$ MuttenSignau (20), 16 = Gondiswil-Ufhusen, $17=$ Dürnten (20), $18=$ Uster (20), $19=$ Katy (10). Abkürzungen: $\mathrm{A}=$ Amsterdam, $\mathrm{B}=$ Berlin, $\mathrm{Be}=\mathrm{Bern}, \mathrm{Br}=$ Bremen, $\mathrm{Bu}=$ Budapest, $\mathrm{F}=$ Frankfurt, $\mathrm{H}=$ Hamburg, $\mathrm{Ha}=$ Hannover, $\mathrm{I}=$ Innsbruck, $\mathrm{K}=$ Kopenhagen, $\mathrm{Kö}=\mathrm{Köln}$, $\mathrm{Kr}=$ Krakau, $\mathrm{Ki}=$ Kiel, $\mathrm{M}=$ München, $\mathrm{P}=$ Prag, Po = Posen, $\mathrm{S}=$ Stuttgart, $\mathrm{Sa}=$ Salzburg, $\mathrm{W}=\mathrm{Wien}, \mathrm{Wa}=$ Warschau, $\mathrm{Z}=$ Zürich.

Bemerkenswerte Abfolgen bieten Selle \& Schneekloth (1965, ergänzt durch Schneekloth 1966) für Oerel, SchütrumpF (1967) für Loopstedt und Geesthacht, Menke (1980) und derselbe in Stremme \& Menke (1980) in den z. T. noch nicht ausführlich publizierten Profilen von Keller und von Rederstall (in Schleswig-Holstein). Das Profil Rederstall ist heute wohl das schönste mehr oder weniger zusammenhängende Profil Nordeuropas, im Florencharakter deutlich stärker nordboreal als Amersfoort in den Niederlanden. 
Eine auf den ersten Blick problematische Angleichung haben wir unumgänglich gefunden: alle Stadiale und Interstadiale stets auf gleiche Tiefenerstreckung umzuzeichnen. Wir haben aber vorgängig die Tiefenerstreckungen annähernd und kritisch gemittelt, da wir ja zur Zeit einen wünschbaren Zeitmaßstab nicht besitzen.

Wir wissen, daß unsere Darstellungen und Auswertungen auf folgenden Voraussetzungen beruhen, die z. T. individuelle Interpretationen sind:

a) Die verglichenen Reihen von Klimaschwankungen 1. Grades sind in jedem Diagramm vollständig.

b) Lokale Schwankungen 2. Grades mögen gelegentlich die Korrelation erschweren, können aber beim heutigen Stand der Forschung noch kaum in Betracht gezogen werden.

c) Die Begrenzung der einzelnen Phasen im Sinn der Schaffung synchroner Abschnitte stellt von Phase zu Phase und von Lokalität zu Lokalität recht schwierige Aufgaben, die persönliche Entscheidungen erfordern und nur in den großen Zügen allgemeine Zustimmung finden werden.

d) Die statistische Basis ist mit unseren 5 Diagrammen für eine vergleichende Auswertung reichlich schmal.

e) Hie und da ist eine Verfälschung der dargestellten Pollenniederschläge durch sekundär eingeschwemmten ältern Pollen nicht völlig auszuschließen (besonders in den mineralischen Sedimenten der Kaltphasen).

Wir gliedern Vergleich und Diskussion in eine allgemeine Charakterisierung und eine spezielle Darstellung des Frühwürms und seiner anspruchsvollen Gehölze.

\section{Allgemeine Charakterisierung des Eem-Interglazials (R/W-Interglazials) und des Frühwürms}

$$
(\mathrm{FW}=\text { Frühwürm und Frühweichsel; } \mathrm{St}=\text { Stadial; Interst. = Interstadial })
$$

Eem-Interglazial: Eine Dreigliederung bietet sich an (wenn man von den einleitenden Betula- und Pinus-Phasen absieht). Der erste, unterste Abschnitt umfaßt extreme EMW (Eichenmischwald)-Werte, dann Corylus-Ausbreitung (früh Ulmus und Fraxinus; lokal starke Alnus-Kodominanz, z. B. in Odderade, Amersfoort und am Sulzberg). Der mittlere Abschnitt ist in Tieflagen gekennzeichnet durch Carpinus-Wälder, in montanen und alpennahen Lagen durch Abies-Wälder mit starkem bis dominantem PiceaAnteil (mehrfach mit stärkerem Taxus-Anteil, lokal auch mit viel Alnus). Im obersten Abschnitt verschwinden die genannten anspruchsvollen Gehölze (abgesehen von Picea und Alnus) fast ganz und werden nach dem Ausweis von Sulzberg-Baden (Diag. 3) zweimal durch eine Dominanzfolge Picea/Pinus mit gelichtetem Waldbestand abgelöst.

Es besteht der Verdacht, daß diese Endphase an vielen Orten Entwicklungshemmungen oder sogar Erosionseinflüssen ausgesetzt war. (Sie scheint jedoch in Grande Pile und in Brörup angedeutet, fehlt aber den Diagrammen von Zeifen und Eurach in Oberbayern, BEug 1976, gänzlich.) Danach ist wahrscheinlich die letzte Warmzeit mit zwei interstadialartigen Picea-Phasen ausgeklungen, die von untypischen Kühlphasen mit geringer Waldlichtung und Pinus-Dominanzen begrenzt waren.

FW-St 1 : ist überall durch mineralische Sedimente stratigraphisch als auch biostratigraphisch als sehr kaltes Stadial erkannt, das jegliche geschlossene Gehölzvegetation vernichtete, selbst in den günstigen Lagen westlich der Vogesen. WeLTEN (1981) supponiert einen sehr kräftigen Gletschervorstoß, der zwar biostratigraphisch bewiesen erscheint, dessen Ausmaß aber geologisch noch nicht erfaßt ist. 
F W - Interst 1, A m e r s f o or t : führt im allgemeinen über Strauchtundra-Phasen zu einer Birken-, ev. Kiefernbewaldung, selten zu Fichtenwäldern, sehr selten zu Nadel-Laub-Mischwäldern. Die Phase ist oft schwer nachzuweisen (infolge fluvioglazialer Störungen) und schwer gegen Brörup abzugrenzen, da sie bei undeutlicher stratigraphischer Abgrenzung als Vorphase von Brörup aufgefaßt werden kann.

FW-St 2 : stellt einen schwachen, wenn auch deutlichen Klimarückschlag dar, der nicht überall zur völligen Vernichtung der Birken-Kiefern-Gehölze führt, in Nord- und Mitteleuropa jedoch die anspruchsvolleren Laubgehölze verdrängt, die Fichte aber in Spuren erhält.

FW-Interst 2, Brörup: scheint das längste und wohl auch wärmste FWInterstadial darzustellen. Es kommt bis an die Nordsee zur zeitweisen Fichtenbewaldung, ev. zu hoher Alnus-Ausbreitung, im westlichen Europa zu vorübergehender Dominanz von Laubgehölzen über Kiefer und Fichte. Die zweite Hälfte ist fast überall Pinus-reich mit Subdominanz von Picea, z. T. von Larix. Larix ist im weit vorgelagerten Alpenvorland spärlich, in Alpennähe und in Nordeuropa stellenweise reichlich vorhanden.

FW-St 3 : ist in minerogenen Sedimenten oft sehr ausgedehnt und scheint einen frühen schwächern und einen spätern intensiven Klimarückschlag aufzuweisen. In organogenen Sedimenten scheint der Rückschlag fast zu fehlen, indem Torfe ihr Wachstum beinahe eingestellt hatten oder anerodiert wurden. Das Stadial hat über längere Zeit (in 300-700 m Höhe) z. T. bloß Waldgrenzverhältnisse aufgewiesen mit Pinus, Pinus cembra, Larix, Betula, Juniperus und wenig Picea, z. T. extramoränisch eine schwache Picea-Dominanz (Grande Pile X 1392,5 cm, Sulzberg $1120 \mathrm{~cm}$, entsprechend dem Ambitzgi-Interstadium, WELTEN 1981).

FW - Interst 3, O d d e r a d e : ist kürzer und kühler als Brörup. Es zeigt in der ersten Hälfte abrupt einsetzende Anteile von anspruchsvollen Gehölzen, in der zweiten Hälfte Pinus-Picea-Dominanzen (in N-Europa nur geringe Picea-Prozente).

FW - S t 4 : setzt mit großer Intensität ein, die alle anspruchsvollen Gehölze, auch Picea, lokal zum Verschwinden bringt. Es weist im Anfang einen Pinus-Nachgipfel des Odderade-Interstadials auf.

FW-Interst 4, Dürnten (sensu Welten 1981): ist sehr kurz und erreicht nur vorübergehend \pm volle Bewaldung mit Pinus und Betula, z. T. mit Picea und viel Larix, doch nur mit Spuren von Laubhölzern.

(F W-St $5=$ ) Mittelwürm-Sta dial 1: Mit diesem Stadial, das vermutlich tiefe kalt-kontinentale Klimawerte erreichte, kann man wahrscheinlich das Frühwürm enden und das Mittelwürm beginnen lassen (sensu Mangerud et al. 1974, Welten 1979).

\section{Spezielle Darstellung des Frühwürms und seiner anspruchsvollen Gehölze in Mittel- und Nordeuropa (Abb. 2-6)}

\section{1.}

Die Rückwanderung anspruchsvoller Gehölze am Beginn der Interglazialphasen ist schon mehrfach Objekt der Bearbeitung und Diskussion gewesen, freilich oft unter Anwendung diskutabler Kriterien. Rückgang und Vernichtung anspruchsvoller Gehölze scheinen keine Probleme zu bieten, solange wir nur schon den Beginn der Würmeiszeit nicht absolut datieren können. Die relativ kurzfristigen Klimaschwankungen des Frühwürmabschnittes der Würmeiszeit (die immerhin Jahrtausende umfassen können) zeigen uns aber klar, daß diese Vernichtung keineswegs ein Sein- oder Nichtsein-Problem ist, sondern ein subtiles lokal- und zeitabhängiges Verdünnungsproblem. Biologisch-physiologisch hat allerdings die Gehölzsippe in ihren Oekotypen angeborene autökologische 
Schwellenwerte, unterhalb welchen sie abstirbt, ausgelöscht wird. Oekologisch bietet aber der Lebensraum einer Gehölzsippe so unterschiedliche Biotope, daß wir bei Klimaverschlechterungen berechtigt sind, von Verdünnung, Verdrängung zu sprechen. Die Stufen der Verdrängung gehen von Lichtung und Konkurrenzierung zu Vorkommen an Spezialstandorten und schließlich zur vielleicht intermittierenden (Anemochorie, Zoochorie!) Auslöschung - in einer bestimmten Gegend. Andere Gegenden stehen nicht in derselben Phase der Verdrängung, da sich die Eiszeitphänomene nach geographischer Breite, Höhenlage, Ozeannähe, atmosphärischen Verhältnissen, Exposition und Bodenunterlage und spezieller Topographie verschieden auswirken. Auslöschung, Reliktstandort und Rückwanderung sind extrem heikle bioklimatische und biogeographische Probleme, die nie in den letzten Grundtatsachen aufzuklären sind, weil sie sich, mathematisch gesprochen, in der Nähe des Nullwertes abspielen.

Um so interessanter ist es, im Bereich Mittel-/Nordeuropa die Wirkungen eines Großversuchs näher zu betrachten, den die Klimaschwankungen im Frühwürm darstellen.

\section{2.}

Wir wagen es, aus unseren vier reproduzierten Diagrammfolgen (und weiteren Beispielen: Odderade, Averdieck 1967, und Osterwanna, Behre 1974, in Nordeuropa, Dürnten und Uster, Welten 1981 [im Druck], und Gondiswil, S. Wegmüller [unpubliziert], am Alpennordrand) folgende Schlüsse zu ziehen und unserer Betrachtung zu Grunde zu legen:

a) Die völlig übereinstimmenden biostratigraphischen Reihen lassen keine Zweifel zu, daß das $\mathrm{R} / \mathrm{W}$-Interglazial im Alpenbereich mit dem Eem-Interglazial in Nordeuropa identisch und synchron ist.

b) Die unterschiedliche Ausbildung der Vegetationsentwicklung in den dargestellten und den oben erwähnten Eem-Interglazialen muß als Tatsache und Beweis dafür genommen werden, daß die Unterschiede echte, z. T. biogeographische, z. T. standörtliche Ausbildungen ein und desselben Interglazials darstellen (und einige weitere Unterschiede auf ungleichem Erhaltungszustand des Einbettungsmittels und des Pollenmaterials beruhen). So weisen die anspruchsvollen Gehölzarten im Eem-Interglazial an den verschiedenen Lokalitäten unserer Vergleichsprofile folgende kleinste und größte Maximalwerte auf:

$\begin{array}{lrlr}\text { EMW } & 9-60 \% & \text { Abies } & 4-52 \% \\ \text { Corylus } & 32-67 \% & \text { Alnus } & 8-57 \% \\ \text { Carpinus } & 6-48 \% & \text { Picea } & 21-74 \%\end{array}$

\section{3.}

Früh schon haben ANDERSEN (1961) und ZAGwiJn (1961) auf die nicht geringen Vorkommen von Pollen wärmeliebender Gehölze in den Frühwürm-Interstadialen hingewiesen, die als Inhalt von organischen Sedimenten nicht anders denn als Fernflugpollen oder Rückwanderungszeugen an der Lokalität zu deuten wären. Diese Ansicht ist durch das regelmäßige Auftreten in andern untersuchten Profilen und durch das verstärkte Auftreten in Wanssum im Moselgebiet (ZAGWIJN 1961) gefestigt worden, wobei man damals vorsichtig und richtig an ökologisch bevorzugte Lokalitäten dachte. Die Untersuchungen am Nordrand der Alpen zeigten dieselbe Erscheinung, mußten aber meistens zurückhaltend beurteilt werden, weil im topographisch bewegten Reliefgebiet der Voralpen immer wieder Pollenverschleppung mit mineralischen Sedimenten festgestellt oder doch vermutet werden mußte. 
1975 publizierte WOILlARD eine völlig überraschende und vorerst unverständliche Diagrammreihe aus dem westlichen Vorgelände der Vogesen (Grande Pile, $330 \mathrm{~m}$ ü. M., Gemeinde St. Germain, im Tal des Ognon im oberen Teil des Flußgebiets der Saône). Auf das Eem-Interglazial folgen drei Warmphasen, die zwar Pinus-, Picea- und Betula-reich sind, doch klare Dominanzen von Corylus, EWM und Carpinus aufweisen und deshalb von der Autorin vorerst als neuentdeckte Interglaziale aufgefaßt wurden. Diese Deutung ist mehrfach bestritten worden (z. B. GRÜGER 1979b). In jüngster Zeit hat sich die Auffassung durchgesetzt, daß es sich bei den Warmphasen von „St. Germain Ia, St. Germain Ic und St. Germain II" der Autorin um die in Nordeuropa nachgewiesenen Interstadialphasen Amersfoort (= Rodebaek ?), Brörup und Odderade handelt. Was auffällig und völlig neu ist an den Ergebnissen von Grande Pile, ist die große Menge der wärmeliebenden Elemente dieser Interstadialphasen gegenüber Zentral- und Nordeuropa.

Unsere 4 Korrelationsdiagramme geben über das Geschehen im Frühwürm (nach C14_ Bestimmungen in Groningen ca. 70'000 bis 55'000 B.P., d. h. vor heute) Auskunft. Wir versuchen, es zu beschreiben.

\section{4.}

Die erste Frühw ürm-Kalt pha s e, die wir hier als FW-St 1 (WeLten 1981: T 1a) bezeichnen, hat in Nord-, Zentral- und Westeuropa die Wälder völlig vernichtet. Bis zu welchem Grad in westlichen und südlichen Randgebieten Europas Pinus-, ev. BetulaWälder mit Anteilen von Laubhölzern vorhanden waren, wissen wir heute noch nicht, halten das aber für sehr wahrscheinlich. In unserem Untersuchungsgebiet (soviel zeigen unsere Vergleichsdiagramme) überlebten Pinus, Betula, Juniperus und spurenweise Alnus und Picea, im westlichen Europa dazu Spuren von Quercus, Ulmus, Fraxinus, Acer, Tilia, Corylus, Carpinus, im östlichen (und südöstlichen) Europa Spuren von Abies. Aus hohen Lagen und von $E$ scheinen schon in dieser Kaltphase Lärchen vereinzelt in unsere Tieflagen eingewandert zu sein.

Es scheint zwingend, daß wir hier einen ersten starken Gletschervorstoß der Würmeiszeit annehmen ( T 1a). Ob und wie weit er ins Vorland hinaus gereicht hat, ob ihm ein bekannter Moränenstand entspricht, wissen wir heute noch nicht. Nach unseren Untersuchungen (WeLTEN 1981) hat er mindestens den Stand der späten Züricher Moränen erreicht, hat aber die Moränen von Killwangen nicht überschritten.

\section{5 .}

Nach seinem Rückzug, der um 70'000 B.P. zu Ende gegangen sein dürfte, breiteten sich nach einer markanten Artemisia-Ephedra-Juniperus-Spätglazialphase (im N Callunaund Empetrum-Heiden) Betula-Pinus-Wälder aus und leiteten ein Interstadial, das Amersfoort-Interstadial, ein mit zunehmender Picea, im N spärlich, am Samerberg und am Sulzberg bis zur Dominanz. Im N erreichten EMW und Alnus Werte von $5 \%$ oder wenig mehr, Corylus solche von $3-5 \%$, während Carpinus-Pollen mit weniger als $1 \%$ doch wohl als Fernflug aufzufassen sind.

Demgegenüber erreichten die EMW-Elemente in Grande Pile 22\%, Carpinus 20\%, Corylus $14 \%$ und dokumentieren dadurch eine typische Ausbreitung und Ansiedlung aus günstigen, nahe gelegenen Refugienstandorten heraus. Es scheint, daß damals Quercus und Acer bis an den östlichen Alpenrand „vordrangen“, Corylus mindestens die schweizerischen Lokalitäten „erreichte“. Wohl möglich, daß sie ihren Ursprung aus geschützten Refugien am Jura-Südrand (oder aus dem Donautal) nahmen und nicht aus dem RhoneSaône-Gebiet oder aus der Oberrheinischen Tiefebene rückwanderten. 


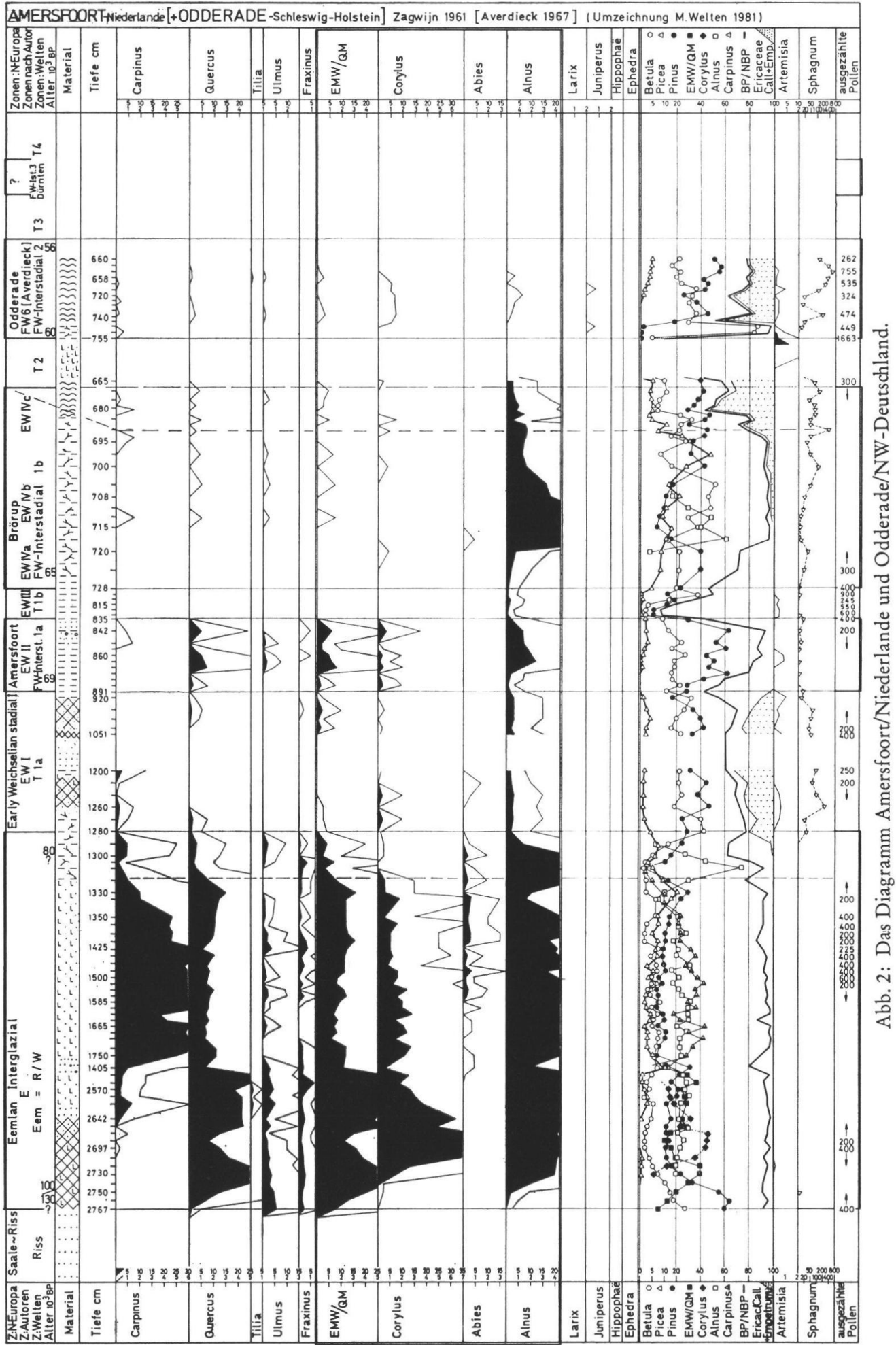




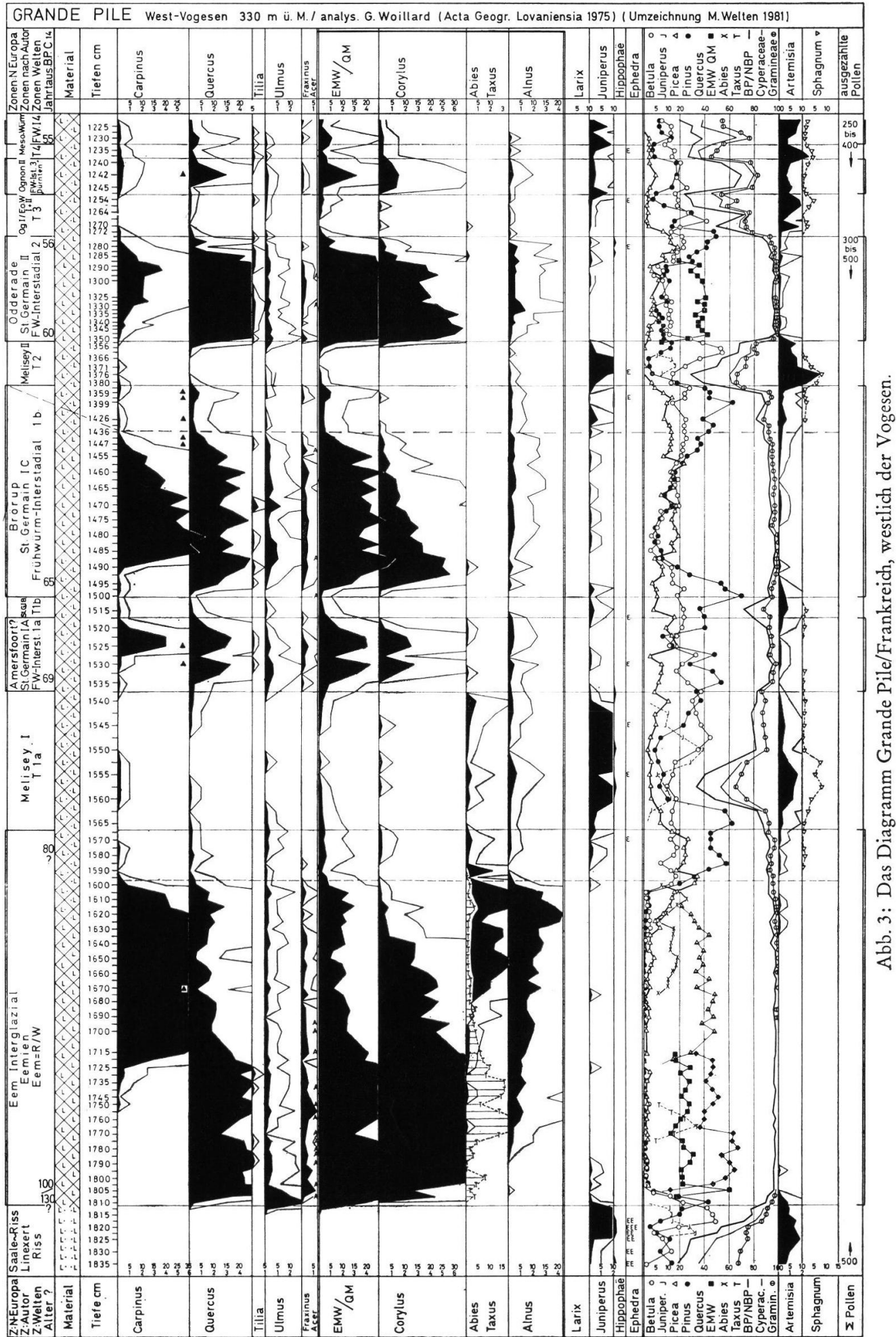




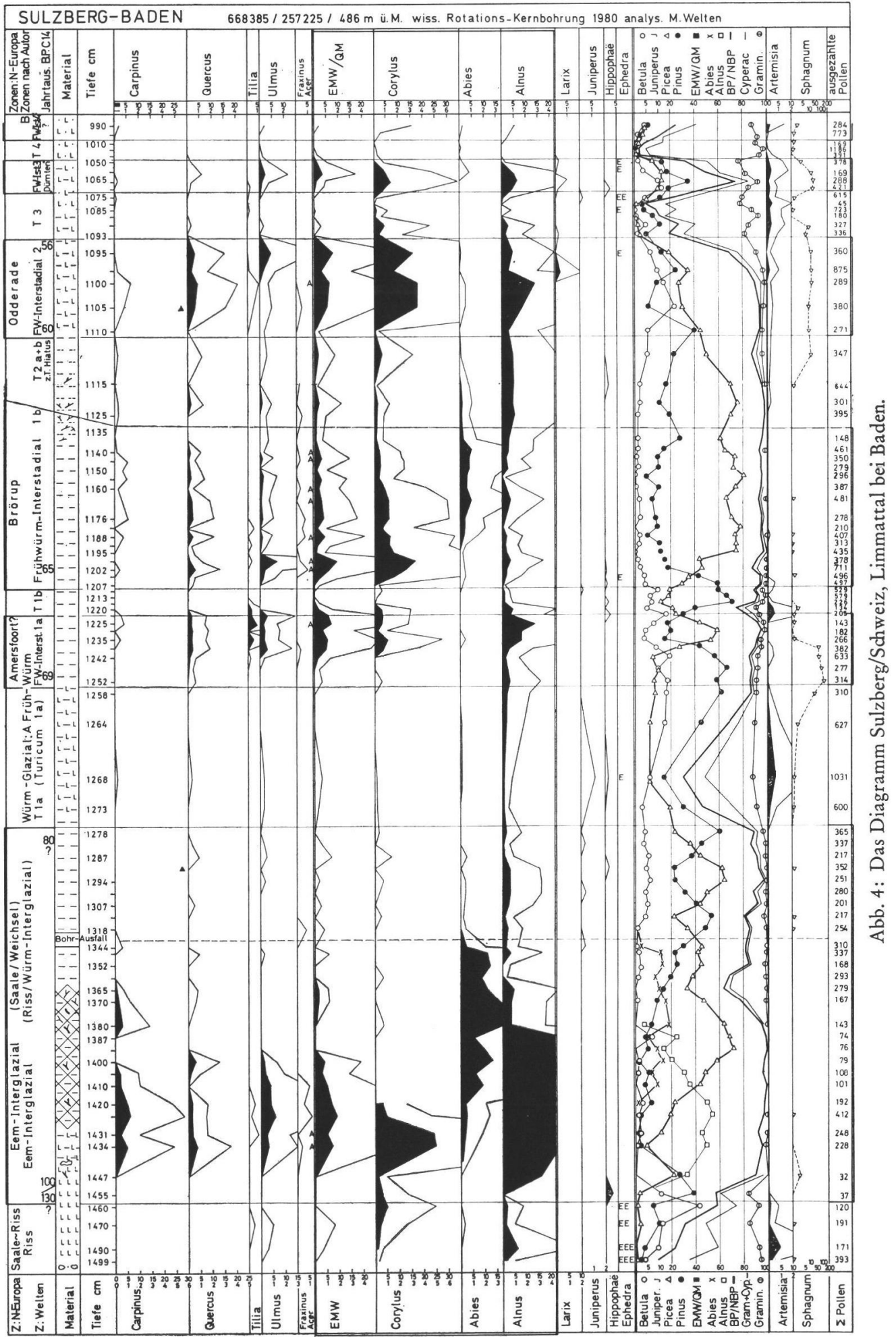




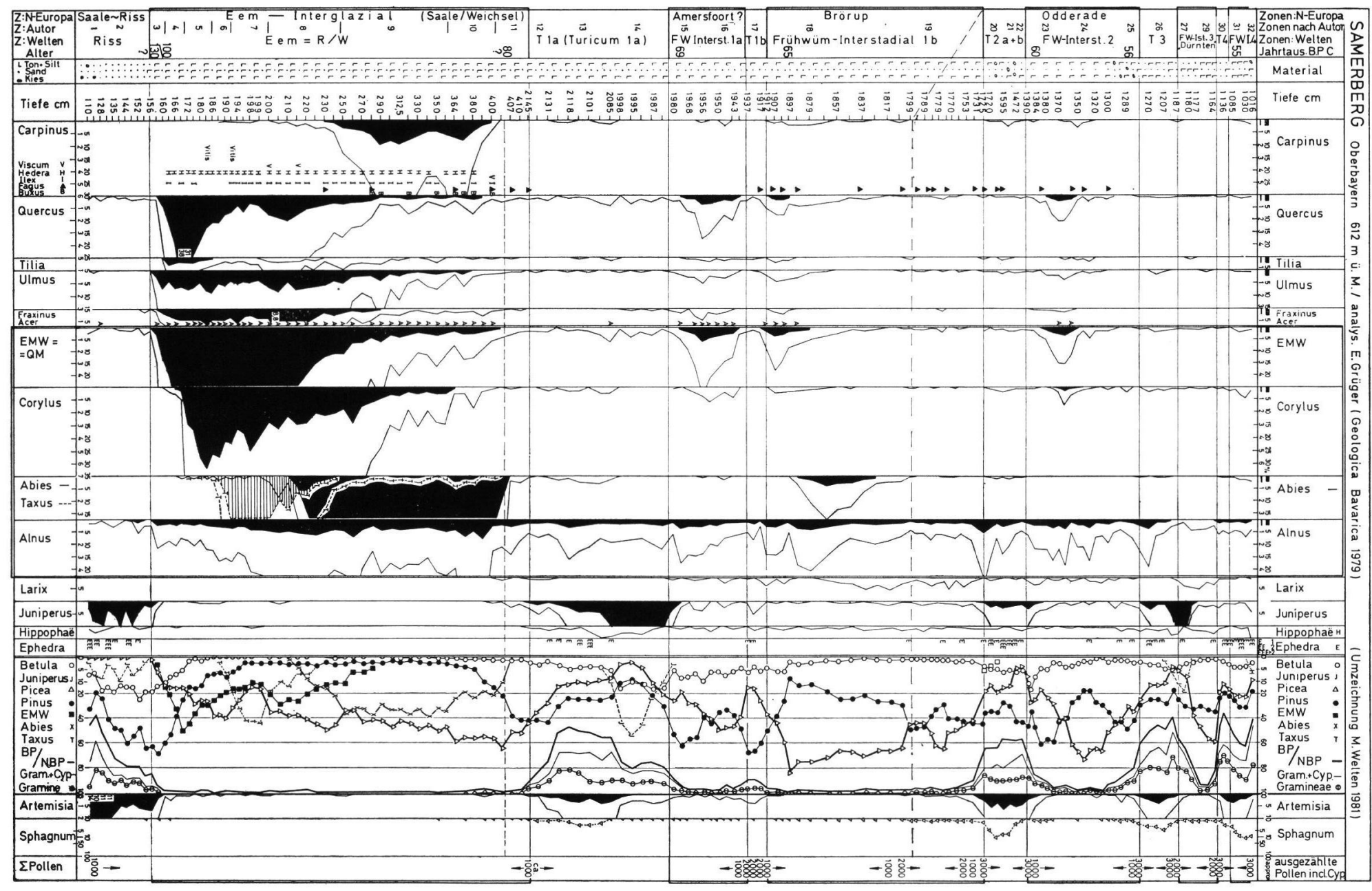

Abb. 5: Das Diagramm Samerberg/S-Deutschland, Oberbayern. 
Die geringe Dauer des Amersfoort-Interstadials und (oder) sein noch recht ungünstiges Klimaoptimum ließen in Zentraleuropa noch kaum über Pinus- und Picea-Wälder hinausgehende Waldvegetation entstehen mit wenigen Laubwaldelementen an besonders günstigen Stellen. Bereits westlich der Vogesen und im Rhonetal bei Lyon (J. L. DE BeAulieu, mdl.) kam es zur Entwicklung von Laub-Nadel-Mischwäldern, immer noch mit starkem borealen Einschlag.

\section{6 .}

Danach vernichtete ein relativ kurzer Klimarückschlag (Gletschervorstoß FW-St2, Welten 1981: T 1b) im N Picea ganz, am Alpenrand aber nicht völlig. Picea blieb hier einzeln oder in Gruppen den lichten Pinus- und Betula-Beständen beigemischt. Auch westlich der Vogesen wurden die anspruchsvolleren Laubwaldelemente verdrängt; Pinus und Betula bildeten lichte Gehölze. Die allerorten schwache Entwicklung spätglazialer Kältesteppenvegetation läßt vermuten, daß der Grad der Abkühlung und Gletscherbildung in dieser Phase weit hinter der FW-St 1-Phase zurückblieb.

\section{7.}

Das nun beginnende $\mathrm{z}$ we ite Frühw ürm-Interstadial, FW-Interst 2, das B rörup-Interstadia l, wird deshalb nirgends mit einer massiven Juniperusund Betula-Phase eingeleitet, sondern sofort mit einer vermehrten Einmischung von Picea und einem Abbau der Betula- und Pinus-Anteile.

Westlich der Vogesen kam es zu einer fast gleichzeitigen explosionsartigen Entwicklung der wärmeliebenden Gehölze, vermutlich aus ihren regional überall vorhandenen Refugien heraus. Nur Carpinus kam zögernd, trotzdem mächtig zur Dominanz. WoILlaRd (1975) verglich die Entwicklung sehr richtig mit den Wiederbesiedlungsvorgängen am Beginn des Holozäns (Postglazials) und des Eem-Interglazials. Wenn wir die noch hohen Prozentwerte von Picea (10-20\%), von Pinus (5-10\%) und von Betula (5-20\%) in Grande Pile mit den entsprechenden und viel niedrigeren im Eem vergleichen (Picea $0-5 \%$, Pinus $1-3 \%$, Betula $0-3 \%$ ) werden wir ihr Brörup ruhig als kühles Interglazial bezeichnen können und den Namen eines Interstadials nur aus Analogie (man möchte biologisch sagen Homologie) zur synchronen Phase in Nord- und Osteuropa und in Alpennähe einer Neuschöpfung einer Bezeichnung für eine intermediäre Ausbildung vorziehen. Schließlich handelt es sich um Chronozonen des würmeiszeitlichen Zeitabschnittes, die in verschiedenen Weltgegenden eben unterschiedlich warm waren (vgl. MaNGerud et al. 1974).

Interessant ist nun, daß sich an den anderen Lokalitäten die erwähnten quasi-Wiederbesiedlungsvorgänge nur (auch verglichen mit dem Amersfoort-Interstadial) sehr schlecht oder auch gar nicht abzeichnen (in Nordeuropa). Das rührt offenbar daher, daß die Wälder in der Kaltphase Amersfoort/Brörup nicht vernichtet, sondern nur gelichtet waren und mit der Erwärmung bloß dichter wurden aus den vorhandenen Elementen heraus: in Nordeuropa aus Pinus und Betula (Picea, Alnus), in Zentraleuropa aus Picea und Pinus heraus. Der imposante Anstieg von Picea am Alpennordrand ist Folge der geschilderten Ausgangslage. Die trotzdem deutlichen, doch schwachen Initialphasen des EMW (incl. Acer) am Samerberg und am Sulzberg können auf Rückwanderung beruhen, ebenso der stärkere Corylus-Anstieg am westlicher gelegenen Sulzberg, z. T. aber auch auf Fernflug, sind aber vielleicht schwache Belebungserscheinungen von regionalen Relikten. Daß sie so rasch abklingen, rührt von der mächtigen Konkurrenz von Picea her (ein Indiz mehr für die Komplexheit der Vorgänge).

Dieser Initialphase des Brörup-Interstadials folgt eine klimatisch vielleicht mildere (weniger kontinentale), vielleicht etwas feuchtere Phase, die dieses vor anderen Früh- 
würm-Interstadialen auszeichnet: Am zentralen Nordalpenrand stellt sich eine längere Phase von Abies ein mit Werten von 5-10\% (in vier Diagrammen deutlich), interessanterweise mit bis über $10 \%$ aber auch in Katy in den Pieninen in den Westkarpaten, vgl. Mamakowa 1975. Dieser Phase scheint in Grande Pile die Carpinus-Phase mit viel Picea zu entsprechen, im N schwankend bald Alnus-, bald Betula-, bald Picea-reiche Abschnitte.

Der letzte Abschnitt des relativ langen Brörup-Interstadials zeichnet sich überall aus durch starke Pinus-Zunahme (und leichte NBP-Zunahme), in den nördlichen und mehr kontinentalen Profilen (auch Katy !) durch schwache bis sehr starke (Einwanderung und) Ausbreitung von Larix (in Grande Pile und Sulzberg nicht festgestellt, doch deutlich in Dürnten im Zürcher Oberland und in Mutten-Signau, Welten 1981). In diesem letzten Abschnitt gehen überall die wärmeliebenden Arten auf geringe Werte zurück.

\section{8 .}

Die dritte Frühwürm-Kaltphase (FW-St. 3, Welten 1981: T2a + b) scheint in ihrem Verlauf recht heterogen. Sie kann sich in Profilen mit vorwiegend mineralischen Sedimenten über mehrere Meter erstrecken, in Profilen mit vorwiegend organischen Materialien auf fast Null verkürzt sein. Im ganzen hatte sie den Charakter einer mäßig kalten, torrentiellen(?), ev. solifluidalen Waldgrenzphase mit starker Pinus-Dominanz (z. T. sicher Pinus cembra), mit geringer Larix-Beimischung und in der ersten Hälfte noch reichlichem Picea-Anteil. Die Kältesteppenvegetation war am Anfang, besonders aber gegen das Ende mittelstark ausgebildet. Gegen Ende scheint sehr verbreitet ein BetulaGipfel aufzutreten (ev. auch vor dem letzten Kältemaximum), doch fehlt überall eine betonte Juniperus-Wiederbewaldungsphase. Das hängt vielleicht mit dem Charakter des FW-St. 3 als Waldgrenzphase zusammen (die ich 1976 und 1978 als Ambitzgi-Interstadium bezeichnete; kalt-kontinental ?).

\section{9.}

Das dritte Frühwürm-Interstadial, FW-Interst 3, das Odderade-I n t e r s t a di a 1 , beginnt sehr früh mit einer kräftigen Corylus-EMW-Alnus-Phase mit regional starker oder schwacher Pinus-Dominanz. Die Summe der genannten anspruchsvollen Gehölze ist größer als am Börup-Beginn, was wohl daher rührt, daß sich die Wälder im Brörup rasch verdichteten, im Odderade aber unter dem Einfluß eines kühleren Interstadialklimas lichter und weniger vital waren (im Norden durchgehende Calluna-Empetrum-Vegetation und geringe Picea-Ausbreitung, in Grande Pile nur schwache CarpinusEntwicklung). Das Odderade-Interstadial war zudem kürzer als das Brörup-Interstadial und weist schon früh gegen das Ende Elemente der Kältesteppe auf, obwohl es in der oberen Hälfte überall starke Picea-Zunahmen zeigt.

\subsection{0.}

Über dem Odderade-Interstadial findet man überall (im $\mathrm{N}$ nicht untersucht) ausgeprägte Kaltphasen mit hohen Kältesteppenelement-Anteilen, die für ein kaltkontinentales Klima sprechen (FW-St. 4). WoIllard hat darin zwei kurze positive Phasen, Ognon I und Ognon II, unterschieden, die sie zwei nordischen Frühweichsel-Interstadialen zuzuordnen versuchte (vgl. WoIllard 1975 und bes. 1978). Ihr Ognon I stellt jedoch nur einen schmalen Pinus-Gipfel dar ohne jede Begleitung von anspruchsvollen Elementen. Er findet sich ebenfalls im Zürcher Oberland und am Samerberg, ebenso merkmalsarm, und muß deshalb vorläufig als kleine Schwankung zweiten Grades unbeachtet bleiben. Viel wichtiger, weil gut charakterisiert, ist Ognon II, das in Mitteleuropa bereits mehrfach nachgewiesen ist (von Welten 1981 als Dürnten-Interstadial bezeichnet). Wir ordnen 
Ognon II unserem FW-Interst. $4 \mathrm{zu}$. Es ist äußerst kurz, erreicht gute Pinus- und PiceaWerte, z. T. hohe Larix-Werte (um 20\%), wird z. T. von hohen Juniperus-Werten eingeleitet, die von ordentlichen Hippophä̈-Werten begleitet sind, was bedeutet, daß die Wiederbewaldung hier von Null an, von offener Kältesteppe an eingesetzt hat (wie am Beginn des Amersfoort-Interstadials). Es weist bis in den westlichen (schweizerischen) Nordalpenraum hinein deutliche Fernflugspuren anspruchsvoller Gehölze auf (die in Grande Pile noch $16 \%$ EMW und $7 \%$ Corylus ausmachen, am Sulzberg 3\%, resp. $6 \%$, also vielleicht selbst am Sulzberg noch ortsanwesend gewesen waren, z. B. an der Lägern). Es scheint nach einer Groninger Datierung an Material aus Dürnten erwiesen, daß diese wahrscheinlich letzte deutliche positive Frühwürmphase wenig älter als 55000 B.P. (GrN9865) ist (WELTEN 1981).

\section{Zusammenfassung}

$\mathrm{R} / \mathrm{W}$-Interglazial im Alpenbereich und Eem-Interglazial in Nordeuropa sind identisch und synchrone Warmzeiten des Jung-Quartärs.

Die Schlußphase dieser letzten Warmzeit dauerte wahrscheinlich länger als bisher angenommen wurde und bestand vielleicht über Jahrtausende im Tiefland Zentraleuropas aus interstadialartiger Pinus- und Picea-Waldvegetation bei bereits stark herabgesetzter Wald- und Schneegrenze und inneralpinem Anwachsen der Gletscher.

Die nachfolgende Vereisung dürfte das Alpenvorland erreicht haben und zu den markanten Eisständen der Würmeiszeit gehören.

Die anschließende Frühwürmphase mag (C 14!) von ca. 70000 bis 55000 B.P. gedauert haben. Sie ist keineswegs eine Phase der progressiven Abkühlung und Herausbildung von Eisschilden und Gletscherströmen, die einem „Maximum“ der Würmvergletscherung entgegen tendierten. Sie stellt im Gegenteil als Ganzes eine mäßige Wärmeschwankung mit vier Interstadialen und drei trennenden Stadialen dar, die auf eine erste große Würm-Vereisungsphase unbekannter Dauer folgten:

Holozän=Postglazial (Warmzeit) 10000 B.P. - heute

Wür m-Eiszeit $=$ Weichsel-Eiszeit

$\begin{array}{lr}\text { Spätwürm } & 25000-10000 \text { B.P. } \\ \text { Mittelwürm } & 55000-25000 \text { B.P. } \\ \text { Frühwürm } & >70000-55000 \text { B.P. }\end{array}$

FW-Stad. 4 kalt

FW-Interstad. 4 Dürnten

ziemlich kühl

FW-Interstad. 3 Odderade mäßig warm

FW-Stad. 3 mäßig kalt

FW-Stad. 2 kühl

FW-Interstad. 2 Brörup warm

FW-Stad. 1 sehr kalt

FW-Interstad. 1 Amersfoort aufwärmend

$\mathrm{R} / \mathrm{W}$ - Interglazia $\mathrm{l}=\mathrm{E}$ em-Interglazia 1

$\begin{array}{ll}\text { R/W-Endphase } & \text { mäßig kühl } \\ \text { R/W-Wärmekulmination } & \text { sehr warm } \\ \text { R/W-Aufwärmphase } & \text { warm }\end{array}$

$\mathrm{R}$ i $\beta-E$ iszeit $=\mathrm{S}$ a a le-Eiszeit 
Mitteleuropa, z. T. auch das südliche Nordeuropa, lagen im Frühwürm im Bereich der Verdrängung, teilweisen Vernichtung und jeweiligen lokalen Wiederausbreitung anspruchsvoller Laubwaldvegetation. In Zentraleuropa dominierten in den Frühwürm-Interstadialen Pinus und Picea, im westlich anschließenden Europa (und wohl auch im südöstlich anschließenden Europa) herrschten im Optimum der Interstadiale Nadel-LaubMischwälder mit borealem Einschlag. In den Stadialphasen fehlte in Mitteleuropa der Wald und regenerierte sich in den Interstadialen aus lokalen Refugien. Eigentliche Wanderungserscheinungen großen Ausmaßes fehlten im Frühwürm weitgehend.

Das völlige (und endgültige ?) Verschwinden von Picea-Wäldern in Zentraleuropa nach dem vierten Frühwürm-Interstadial dürfte den Beginn des Mittelwürmabschnittes markieren.

Vermutlich spielten sich die im Frühwürm beschriebenen Verdrängungs- und Vernichtungserscheinungen der anspruchsvollen Waldvegetation im Mittel- und Spätwürm in sehr ähnlicher Weise in graduell erweitertem Rahmen ab, der Basis für die spät- und postglaziale Wiederbesiedlung war, die aus einem noch stärker aufgelockerten Refugiennetz heraus erfolgte.

\section{Schriftenverzeichnis}

1 Andersen, S. T. (1961): Vegetation and its Environment in Denmark in the Early Weichselian Glacial (Last Glacial). - Danmarks Geol. Unders., II. R., 75: 175 S.; Kopenhagen.

2 Averdieck, F. R. (1967): Die Vegetationsentwicklung des Eem-Interglazials und der Frühwürm-Interstadiale von Odderade (Schleswig-Holstein). - Fundamenta, R. B. 2: 101125; Köln/Graz.

3 Beнre, K. E. (1974): Die Vegetation im Spätpleistozän von Osterwanna/Niedersachsen. Geol. Jb., A 18: 3-48; Hannover.

4 Beug, H. J. (1972): Das Riß/Würm-Interglazial von Zeifen, Landkreis Laufen a. d. Salzach. - Bayr. Akad. Wiss., Math. Naturw. Kl., N. F., 151: 46-75; München.

5 - (1976): Diagramme Zeifen und Eurach a) in Führer DEUQUA-Tagung Stuttgart 1972, b) in Führer Exkursionstagung IGCP-Projekt 73/1/24, herausg. von B. FrenzeL (1976 u. 1978); Bonn.

6 Frenzel, B. (1968): Grundzüge der pleistozänen Vegetationsgeschichte Nord-Eurasiens. 326 S.; Wiesbaden (Steiner).

7 Grüger, E. (1979a): Spätriß, Riß/Würm und Frühwürm am Samerberg in Oberbayern ein vegetationsgeschichtlicher Beitrag zur Gliederung des Jungpleistozäns. - Geologica Bavarica, 80: 5-64; München.

$8-(1979 b)$ : Die Seeablagerungen vom Samerberg/Obb. und ihre Stellung im Jungpleistozän. - Eiszeitalter u. Gegenwart, 29: 23-34; Hannover.

9 KLAuS, W. (1975): Das Mondsee-Interglazial, ein neuer Florenfundpunkt der Ostalpen. Jb. Oberösterr. Musealverein, 130, I. Abh., 315-344; Linz.

10 Мамлкоwa, K. Моoк, W. G. \& Srodon, A. (1975): Late Pleistocene Flora at Katy (Pieniny Mts., West Carpathians). - Acta Palaeobotanica, XVI (2): 147-172; Krakow.

11 Mangerud, J., Anderson, S. T., Berglund, B. E. \& Donner, J. J. (1978): The subdivision of the Quaternary of Norden: a discussion. - Boreas, 7: 179-181; Oslo.

12 Menke, B. (1980): Vegetationskundlich-ökologisches Modell eines Interglazial-Glazial-Zyklus in Nordwestdeutschland. - Phytocoenologia, 7: 100-120; Stuttgart/Braunschweig.

13 - \& Behre, K. E. (1973): History of Vegetation and Biostratigraphy. - Eiszeitalter u. Gegenwart, 23/24: 251-267; Ohringen/Württ.

14 SChNeEKLотн, H. (1966): Ergebnisse weiterer Untersuchungen an den interstadialen Ablagerungen der Weichsel-Eiszeit in Oerel, Krs. Bremervörde. - Z. dt. geol. Ges., 116: $773-$ 796; Hannover. 
15 SchütrumpF, R. (1967): Die Profile von Loopstedt und Geesthacht in Schleswig-Holstein. Fundamenta, B 2: 136-167; Köln/Graz.

16 Selle, W. \& Schneekloth, H. (1965): Ergebnisse einer Kernbohrung in Oerel, Krs. Bremervörde; drei Interstadiale über Ablagerungen des Eem-Interglazials. - Z. dt. geol. Ges., 115: 109-117; Hannover.

17 Stremme, H. E. \& Menke, B. (1980): Quartär-Exkursionen in Schleswig-Holstein. (Rederstall und Keller). - Geol. Landesamt Schleswig-Holstein, S. 41-49; Kiel.

18 Welten, M. (1976): Das Jüngere Quartär im nördlichen Alpenvorland der Schweiz auf Grund pollenanalytischer Untersuchungen. - In: Führer zur Exkursionstagung des IGCP-Projektes 73/1/24 (Herausg. B. FrenzeL), Stuttgart-Hohenheim / 2. Aufl. Bonn, 1978.

19 - (1979): Late Glacial and Late Weichselian (Spätglazial und Spätwürm): a comment. Boreas, 8: 396; Oslo.

20 - (1981): Pollenanalytische Untersuchungen im Jüngeren Quartär des nördlichen AlpenVorlandes der Schweiz. - Beitr. z. Geol. Karte der Schweiz; Basel. - [Im Druck.]

21 Wolllard, G. (1975): Recherches palynologiques sur le Pleistocène dans l'Est de la Belgique et dans les Vosges Lorraines. - Acta Geogr. Lovaniensia, 14: 118 S.; Louvain-la-Neuve.

22 - (1979): The last Interglacial-Glacial cycle at Grande Pile in northeastern France. Bull. Soc. belge de Geol., T 88: 51 -69; Brüssel.

23 ZAGwIJN, W. H. (1961): Vegetation, Climate and Radiocarbon Datings in the Late Pleistocene of the Netherlands. - Mem. Geol. Found. Netherlands, 14: 15-45; Haarlem.

24 - \& PAEPE, R. (1968): Die Stratigraphie der weichselzeitlichen Ablagerungen der Niederlande und Belgiens. - Eiszeitalter u. Gegenwart, 19: 129-146; Öhringen/Württ.

Manuskript eingegangen am 25. 6. 1981. 